\title{
EVALUATION OF A COMPUTERISED IMAGE ANALYSER FOR STUDYING ALTERATIONS IN RADIOGRAPHIC BONE DENSITY IN THE RAT
}

\author{
Elmer E. Specht, Portland, Oregon, United States of America \\ From the Division of Orthopaedics and Rehabilitation, University of Oregon, Portland
}

\begin{abstract}
A computerised image analyser has been used experimentally to determine its possible usefulness in quantifying bone density. Rats were prepared for this purpose and ash weight/volume readings were obtained on femora which had been radiographed and studied to estimate radiographic density by means of the image analyser. The findings indicated that rats can be prepared by administering oestradiol or by allowing an additional week's growth, both of which give a statistically significant increase of bone density. The computerised image analyser was able to detect these differences but the correlations, although real and linear, between ash weight/volume and radiographic density were not large. Further refinements in techniques will be needed before the system is applied clinically.
\end{abstract}

The purpose of this study was to evaluate a computerised image analyser $\left(\mathrm{I}_{2} \mathrm{~S}\right.$ Digicol Model 401$)$ as a possible method of measuring bone density, a use which has been proposed in this journal (Dickson, Paice and Calnan 1973). Animal models were chosen in order to obtain quantifiable data for analysis. The equipment is claimed by its manufacturers to be capable of converting the various shades (tone gradients) of radiographs (or any image) into eight arbitrarily assigned colours, each colour representing one degree of density of the image examined. The manufacturer states: "Each small change in the gradient, whether in black and white or colour, is extracted, converted to digital form and replaced ... in a new image. Each change in tone level is coded in a false colour to sharply differentiate each tone from those surrounding it". It is apparent that this system adds no new evidence but acts upon the interface between the image and the human optical system.

\section{MATERIALS AND METHODS}

Sixty-six male Wistar rats, weighing between 180 and 200 grams and aged forty-four days at the beginning of the study, were divided into four groups corresponding to different treatments. The animals in Group 1 (twenty-two rats) were treated with intramuscular injections of 0.1 cubic centimetre of oestradiol (Delestrogen, Squibb) administered at weekly intervals for a total of four injections. This has been demonstrated to increase bone density in this species. Group 2 (eleven rats) was a control group of animals born on the same day, fed and housed in the same manner, killed on the same day (age seventy-five days) and radiographed with the same technique, which will be described below. The animals in group 3 (twenty-two rats) were fed Purina Chow mixed with beta-amino propionitrile and were killed simultaneously with rats of the same age (eighty-two days) used as controls (Group 4-eleven rats). The thickness of the soft tissues of the left thigh was measured with a caliper and each rat was weighed.

Immediately after death, each rat was radiographed by a standard technique which was not varied throughout the study. An aluminium step-cut wedge was placed immediately to the left of the animal, which was spread-eagled directly upon a standard $\mathrm{x}$-ray casette. Positioning of each animal was constant, as was tube-to-object and tube-to-film distance and the relative positions of the tube, animal and film.

The left and right femurs were then excised. The rats were kept frozen while not being worked on. A solution of 1 gram of trypsin to 20 millilitres of distilled water was used to dissolve soft tissues remaining attached to the femur following excision. This, along with scraping with a surgical scalpel, was used to clean the femora. Calipers were used to measure the length of the left femora the volumes of which were determined by water displacement in a plastic syringe with gradient markings of $\mathbf{0 . 2}$ millilitre.

The left femora were ashed in prepared crucibles for twenty-four hours at $\mathbf{5 0 0}$ degrees Celsius. The crucibles were placed in a desiccating jar to cool and were then weighed. The weight of the ashed bones was determined by taking the difference between the crucible weight with the bone and without the bone. The ash weight thus obtained was divided by the volume to give an approximation of a density measurement. This is referred to as the ash weight/volume measurement.

In the process of cleaning the femora, it was noted that in the group which had been treated with beta-amino propionitrile, the epiphyses slipped off in several individuals, as would be expected (lathyritic effect). This was not felt to influence the accuracy of the ash weight inasmuch as the epiphyses along with the shafts of the bones were ashed and weighed in toto.

The computerised image analyser (Figs. 1 to 3 ) was used to estimate the radiographic density of the left femur of the sixty-six rats. It was found that certain variables affected the image obtained on the monitor screen of the image analyser. These variables required standardisation of technique: 1) the position of the $x$-ray image with respect to the lens of the 


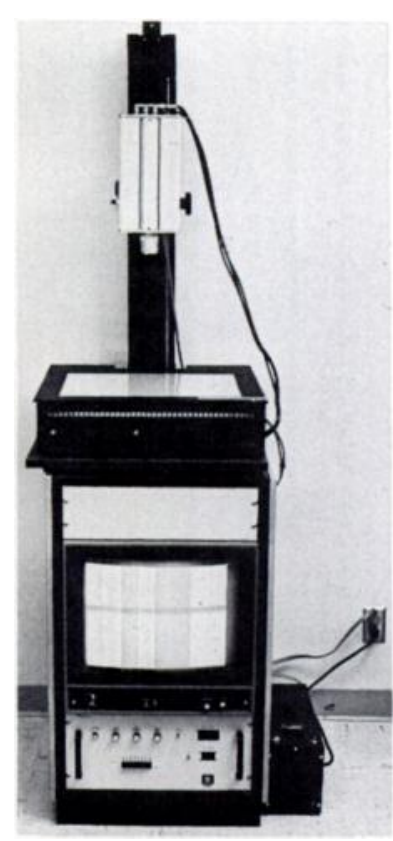

Fici. 1

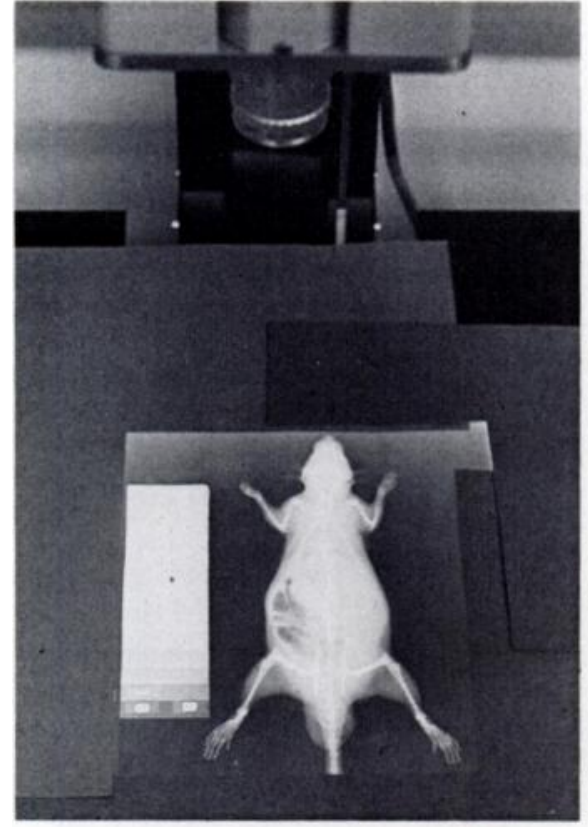

FiG. 2

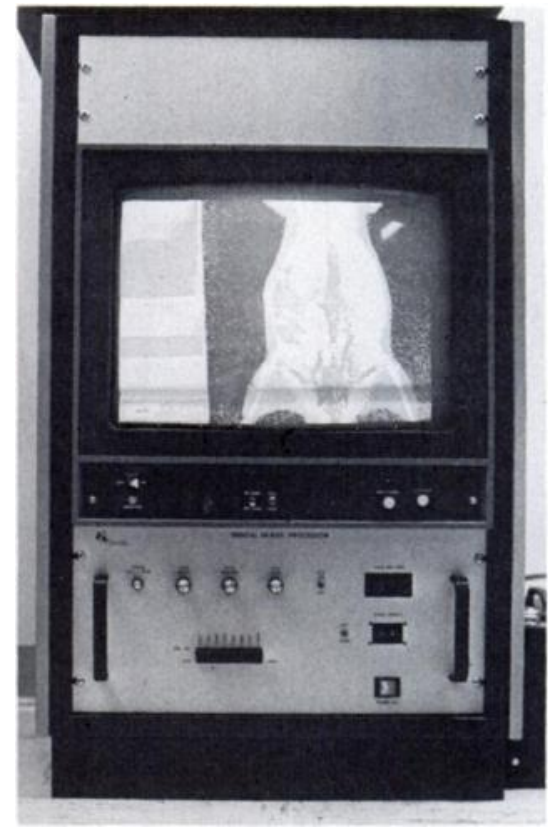

Fici. 3

Figure 1-The computerised image analyser. The essential components, from top to bottom, are the Vidicon electronic scanning camerd. mounted on an adjustable stand, a light box for displaying the image to be studied, a colour television monitor, and a digital computer. Figure 2-The radiograph of a rat is displayed here on the light box beneath the Vidicon camera. Figure 3-The tube of the television monitor displays the computerised image in an eight-colour spectrum. Each arbitrarily assigned colour represents one of eight degrees of radiographic density on the original film. The digital computer below can be tuned to vary the width and the position of each density-co!our on the monitor. The percentage area of each colour visible is also available.

Vidicon scanning camera; 2) the distance between the image and the objective lens of the camera. This distance was maintained constant at an arbitrarily selected 34 centimetres; 3 ) the lens aperture of the Vidicon camera. This was held constant $(f / 5 \cdot 6)$. The aluminium step wedge exposed on the radiographic film with the rats was used to standardise the density colour assignment with the eight colours so adjusted by means of the "slice width" and "slice position" controls as to cause each of the eight thinnest steps of the wedge to be a different colour. The image analyser data for each rat is given as the average radiographic density of the left femur at a point 5 millimetres from the epiphysial line toward the centre of the shaft. The colour at this standardised area was noted by visual observation and a semi-quantitative value was assigned according to the radiographic density of the image as analysed by the computer and displayed on the monitor. The sequence of colours is arbitrarily established at the time of manufacture of the instrument. This sequence is blue, yellow, green, orange, red, brown, violet and magenta proceeding from the least to the most "dense". Blue was arbitrarily assigned the number 1 and magenta the number 8 for purposes of obtaining subsequent statistical correlations. The other colours were assigned the intervening numbers in order. This provided a semi-quantitative measurement of an arbitrarily chosen area of each femur.

\section{RESULTS}

The data thus obtained were then submitted for statistical analysis (Scheffé 1964) with the assistance of a computer. It was found that the oestradiol-treated rats had a significantly elevated ash weight/volume compared to untreated controls of a similar age ( $t$ test $\mathrm{p}<0.01)$ whereas the beta-amino propionitrile group did not differ significantly from its control group. It is postulated that this may be a consequence of inadequate dosage. The ash weight/volume ratio of the oestrogentreated group did differ significantly from that of the beta-amino propionitrile treated group. Scheffé's comparison test $\mathrm{p}<0.01$.

Radiographic density as determined by the image analyser was then compared for each group in the same manner. Both the oestrogen-treated and the betaamino propionitrile treated groups had statistically significant differences from their respective controls

TABLE 1

Ash Weight/Volume Relailid io Radiographic Densities F-Test or Rigirission

\begin{tabular}{|c|c|c|}
\hline Treatment & df & MS reg./MS res. $=\mathbf{F}$ \\
\hline 1 & 1,20 & $5 \cdot 50955^{*}$ \\
2 & 1,9 & 1.50689 \\
3 & 1,20 & 0.25232 \\
4 & 1,9 & $2 \cdot 20165$ \\
\hline All (66) & 1,64 & $13.48831^{*}$ \\
\hline
\end{tabular}

$*$ a statistically significant difference $(p<01)$

( $t$ test $p<0.05$ and $p<0.01$ respectively). The difference between the oestrogen-treated group and the propionitrile-treated group was also significant (Scheffé's multiple comparison test $p<0.01$ ). The two control groups, different in age by one week, also had statistically significant differences between them. These 
differences in radiographic density were not visible to the unaided eye in the black and white radiographs, but only in the computer-analysed image.

Further analysis of the evidence obtained revealed that the correlation between the ash weight/volume ratio and the radiographic density determined by the image analyser and converted to ridits, although it is linear and real (as determined by the F-test of regression - Table I), is not great, a correlation coefficient ( $\mathrm{r}$ in Table II) of 0.41721 obtaining for all animals studied. The best intra-group correlation coefficient, 0.46473 , was obtained for Group 1 (oestradiol-treated) and the worst, $\mathbf{0} \cdot 11162$, for Group 3 (beta-amino propionitrile treated) animals. No explanation for this difference between groups is apparent and it probably represents chance variation.

\section{CONCLUSIONS}

1. Rats can serve as satisfactory animal models for radiographic studies of bone density alterations.

2. As demonstrated by other workers, bone density can be significantly increased by treatment with oestradiol, and also by allowing a week's additional growth.

3. Beta-amino propionitrile, in a dose of $0 \cdot 1$ per cent of the feed consumed, did not alter bone density (ash weight/volume) significantly in this study as compared to a control group.
TABLE II

Ash Weight/Vol.cme $=y$; Radiographic Density (IN Ridits) $=x$

\begin{tabular}{|c|c|c|c|}
\hline Group & $\mathbf{r}$ & $\mathbf{y}=\mathbf{a}+\mathbf{b x}$ & Residual M.S. \\
\hline 1 & 0.46473 & $\mathbf{y}=0.61798+0.086709 x$ & 0.00258490795 \\
2 & 0.37870 & $y=0.57627+0.091020 x$ & 0.00134294444 \\
3 & 0.11162 & $y=0.59916+0.023425 x$ & 0.00260747598 \\
4 & 0.44333 & $y=0.59052+0.075294 x$ & 0.00120001720 \\
\hline All & 0.41721 & $y=0.59095+0.084278 x$ & 0.00248333522 \\
\hline
\end{tabular}

4. The use of a computerised image analyser to evaluate radiographic density of the prepared rat bones did yield statistically significant differences between groups with different bone densities (ash weight/volume ratios), but the correlations, although linear and real, are not great. 5. The computerised image analyser has possible usefulness in measuring radiographic density, but not without major refinements in either the technique of taking the radiographs to be studied (effects of softtissue variation, position of bone, and probably also variations in the energy produced by the $x$-ray tube and developing technique) and the precision of the analyser itself.

6. There is a need for further studies utilising computerised image analysers before results sufficiently accurate for medical uses can be proposed.

The assistance of $\operatorname{Dr~K.~H.~Lu~in~statistically~analysing~the~data~obtained~is~gratefully~acknowledged.~This~study~was~funded~in~large~measure~by~}$ the Shriners Hospitals for Cripp!ed Children and was administered by the Portiand, Oregon, Unit.

\section{REFERENCES}

Dickson, R. A., Paice, F., and Calnan, J. S. (1973) The quantitative colour television image analyser. Journal of Bone and Joint Surgery, 55-B, 359-368

Scheffé, H. (1964) Analysis of Variance. Fourth edition. New York: John Wiley and Sons. 